\title{
Diabetic foot osteomyelitis
}

\author{
Mesut Mutluoglu MD, Benjamin A. Lipsky MD
}

\section{Early manifestations of diabetic foot osteomyelitis are nonspecific}

Infection should be suspected in any patient with diabetes who presents with pain, tenderness, redness, warmth or induration of the foot, especially if an open wound is present. ${ }^{1}$ Bone infection can occur by contiguous spread from soft tissue. ${ }^{2}$

Blood tests and imaging studies support the diagnosis of osteomyelitis

Measuring leukocyte counts or C-reactive protein levels are not useful, but an erythrocyte sedimentation rate greater than $70 \mathrm{~mm} / \mathrm{hr}$ supports the diagnosis of osteomyelitis. ${ }^{1}$ Initial and/or sequential plain $\mathrm{x}$-rays are helpful in diagnosis and monitoring. ${ }^{1}$ Advanced imaging ${ }^{1}$ is required for early diagnosis, delineation of deep soft tissue infection, differentiation of osteomyelitis from neuroarthropathy or to plan surgery. Magnetic resonance imaging (MRI) is preferred; however, radionuclide scanning can be used if MRI is unavailable or contraindicated. ${ }^{1}$ An aseptically collected bone sample for culture and histopathology is the criterion standard for diagnosing osteomyelitis. ${ }^{1}$

CMAJ invites submissions to "Five things to know about ..." Submit manuscripts online at http://mc.manuscriptcentral. com/cmaj

\section{Several clinical findings raise suspicion of osteomyelitis}

Findings suggestive of osteomylitis include: visible exposed bone; bone palpable with a blunt metal probe; a red, swollen ("sausage") toe, especially with an ulcer; an ulcer that is deep, fails to heal or is located over a bony prominence; and the presence of a soft tissue sinus, especially with purulent discharge. ${ }^{1}$

\section{Selected cases may be managed without surgical treatment}

Recent guidance suggests that some cases, especially uncomplicated forefoot infections, may respond to treatment with antibiotics alone. ${ }^{1,3}$ Empiric antibiotic regimens, based on the likely causative pathogens (Staphylococcus aureus is always included), are tailored according to culture results from specimens (preferably of bone). Treatment length is six weeks but can be shorter if infected bone is resected. ${ }^{1,3}$ There is no evidence to support the use of adjunctive therapies, including hyperbaric oxygen. ${ }^{4}$ Patients should be monitored for resolution of clinical signs and symptoms, abnormal laboratory tests and imaging studies (i.e., for bone regeneration) for at least one year. ${ }^{3}$ Patients who do not improve should be evaluated by an interdisciplinary team that includes a surgeon. ${ }^{3}$

\section{References}

1. Lipsky BA, Aragón-Sánchez J, Diggle M, et al.; International Working Group on the Diabetic Foot. IWGDF guidance on the diagnosis and management of foot infections in persons with diabetes. Diabetes Metab Res Rev 2016;32(Suppl 1):45-74.

2. Lew DP, Waldvogel FA. Osteomyelitis. Lancet 2004;364:369-79.
Foot care education is important for prevention

Diabetic foot osteomyelitis can usually be prevented by optimizing footwear and foot care (to avoid breaks in the skin), and treating any wounds to the foot quickly. ${ }^{5}$

3. Lipsky BA, Berendt AR, Cornia PB, et al. 2012 Infectious Diseases Society of America clinical practice guideline for the diagnosis and treatment of diabetic foot infections. Clin Infect Dis 2012; 54:e132-73

4. Peters EJ, Lipsky BA, Aragón-Sánchez J, et al. Interventions in the management of infection in the foot in diabetes: a systematic review. Diabetes Metab Res Rev 2016;32(Suppl 1):145-53.

5. Bus SA, Netten JJ, Lavery LA, et al. IWGDF Guidance on the prevention of foot ulcers in atrisk patients with diabetes. Diabetes Metab Res Rev 2016;32(Suppl 1):16-24.

Competing interests: None declared.

This article has been peer reviewed.

Affiliations: Department of Underwater and Hyperbaric Medicine, (Mutluoglu) GATA Haydarpasa Teaching Hospital, Istanbul, Turkey; Division of Medical Sciences (Lipsky), Green Templeton College, University of Oxford, Oxford, UK

Correspondence to: Mesut Mutluoglu, drmutluoglu@gmail.com

CMAJ 2016. DOI:10.1503/cmaj.160228 